\title{
Elliptic Matrix Representations of Elliptic Biquaternions and Their Applications
}

\author{
Kahraman Esen Özen* and Murat Tosun \\ (Communicated by Yusuf Yayli)
}

\begin{abstract}
In this study, we obtain the $4 \times 4$ elliptic matrix representations of elliptic biquaternions with the aid of the left and right Hamilton operators. Afterwards, we show that the space of $4 \times 4$ matrices generated by left Hamilton operator is isomorphic to the space of elliptic biquaternions. Then, we study the De-Moivre's and Euler formulas for the matrices of this matrix space. Additionally, the powers of these matrices are obtained with the aid of the De-Moivre's formula.
\end{abstract}

Keywords: Complex quaternions; Hamilton matrices; Matrix representations; Elliptic biquaternions.

AMS Subject Classification (2010): Primary: 11R52 ; Secondary: 15B99;

\section{Introduction}

The set of quaternions was introduced by Sir W. R. Hamilton in 1843 [1]. The set of quaternions can be represented as

$$
H=\left\{q=q_{0}+q_{1} \mathbf{i}+q_{2} \mathbf{j}+q_{3} \mathbf{k}: q_{0}, q_{1}, q_{2}, q_{3} \in \mathbb{R}\right\}
$$

where the quaternionic units $\mathbf{i}, \mathbf{j}$ and $\mathbf{k}$ satisfy

$$
\mathbf{i}^{2}=\mathbf{j}^{2}=\mathbf{k}^{2}=-1, \quad \mathbf{i j}=-\mathbf{j i}=\mathbf{k}, \quad \mathbf{j} \mathbf{k}=-\mathbf{k j}=\mathbf{i}, \quad \mathbf{k i}=-\mathbf{i} \mathbf{k}=\mathbf{j} .
$$

The real quaternion algebra has an important place in kinematic, differential geometry, quantum physics and image processing etc.

We know that real quaternion algebra is isomorphic to a real $4 \times 4$ matrix algebra. The real matrix representations of quaternions are investigated in [2, 3, 4]. On the other hand, the Euler's and De-Moivre's formulas for the complex numbers are generalized to quaternions in [5]. These formulas are investigated for real matrices associated with real quaternions in [6].

W. R Hamilton also introduced the complex quaternion algebra within ten years of the discovery of quaternions [7]. Complex quaternion algebra $H_{\mathbb{C}}$ is a generalization of real quaternion algebra by permitting $q_{0}, q_{1}, q_{2}$ and $q_{3}$ to be complex numbers where $\mathbf{i}, \mathbf{j}$ and $\mathbf{k}$ are exactly the same in real quaternions. That is, the set of complex quaternions can be represented as

$$
H_{\mathbb{C}}=\left\{Q=Q_{0}+Q_{1} \mathbf{i}+Q_{2} \mathbf{j}+Q_{3} \mathbf{k}: Q_{0}, Q_{1}, Q_{2}, Q_{3} \in \mathbb{C}\right\} .
$$

Euler formula and De-Moivre's formula for complex quaternions are discussed by M. Jafari in [8]. M. Jafari in this study, also give these formulas for $4 \times 4$ complex matrices generated by left Hamilton operator. Hamilton operators have an important role in the quaternion theory. Some algebraic properties of Hamilton operators are taken into consideration in [9]. Also, some studies which are performed by using the Hamilton operators can be found in $[10,11,12,13]$.

Recently, we have presented the elliptic biquaternions and investigated the terms; inverse, inner product, seminorm, modulus and conjugate for them in [14]. Also, some properties of elliptic biquaternions relating to seminorm and the three types of conjugate have been given and the idempotent elements in the elliptic biquaternion 
algebra have been discussed in [15]. Lastly, some algebraic relations between elliptic biquaternions and extended $p$-trigonometric functions have been discussed in [16].

In this paper, firstly, we present an introduction to elliptic biquaternions and recall some information on elliptic matrices for the necessary background. Afterwards, with the help of the Hamilton operators, we obtain $4 \times 4$ elliptic matrix representations of elliptic biquaternions. Also, we show that the matrix space, which includes the matrix representations corresponding to left Hamilton operator, is isomorphic to the space of elliptic biquaternions. Then, we call the matrix, which corresponds to an elliptic biquaternion $Q$ under this isomorphism, as the elliptic matrix associated with $Q$. Finally, we express the De-Moivre's and Euler Formulas for these matrices by writing them in the $p$-trigonometric form and obtain the powers of these matrices by means of the De-Moivre's formula.

Throughout this paper, the following notations are used. Let $\mathbb{C}_{p}, H \mathbb{C}_{p}$ and $M_{m \times n}\left(\mathbb{C}_{p}\right)$ denote the elliptic number field, the elliptic biquaternion algebra and the set of all $m \times n$ elliptic matrices, respectively.

\section{Preliminaries}

In this section, firstly, we recall the fundamental concepts of elliptic biquaternions (see [14] and [16] for more details.)

The set of elliptic biquaternions is given in the cartesian form as in the following

$$
H \mathbb{C}_{p}=\left\{Q=A_{0}+A_{1} \mathbf{i}+A_{2} \mathbf{j}+A_{3} \mathbf{k}: A_{0}, A_{1}, A_{2}, A_{3} \in \mathbb{C}_{p}\right\}
$$

where $\mathbb{C}_{p}=\left\{x+I y: x, y \in \mathbb{R}, I^{2}=p<0, p \in \mathbb{R}\right\}$ states the system of elliptic numbers and $\mathbf{i}, \mathbf{j}$ and $\mathbf{k}$ are the quaternionic units which satisfy

$$
\mathbf{i}^{2}=\mathbf{j}^{2}=\mathbf{k}^{2}=-1, \quad \mathbf{i j}=-\mathbf{j i}=\mathbf{k}, \quad \mathbf{j k}=-\mathbf{k j}=\mathbf{i}, \quad \mathbf{k i}=-\mathbf{i k}=\mathbf{j} .
$$

For any two elliptic biquaternions $Q=A_{0}+A_{1} \mathbf{i}+A_{2} \mathbf{j}+A_{3} \mathbf{k}$ and $R=B_{0}+B_{1} \mathbf{i}+B_{2} \mathbf{j}+B_{3} \mathbf{k}$, addition and multiplication by a scalar $\lambda \in \mathbb{C}_{p}$ are defined as

$$
\begin{aligned}
Q+R & =\left(A_{0}+B_{0}\right)+\left(A_{1}+B_{1}\right) \mathbf{i}+\left(A_{2}+B_{2}\right) \mathbf{j}+\left(A_{3}+B_{3}\right) \mathbf{k} \\
\lambda Q & =\left(\lambda A_{0}\right)+\left(\lambda A_{1}\right) \mathbf{i}+\left(\lambda A_{2}\right) \mathbf{j}+\left(\lambda A_{3}\right) \mathbf{k} .
\end{aligned}
$$

and also, the product of $Q$ and $R$ is given as follows:

$$
\begin{aligned}
Q R & =\left[\left(A_{0} B_{0}\right)-\left(A_{1} B_{1}\right)-\left(A_{2} B_{2}\right)-\left(A_{3} B_{3}\right)\right]+\left[\left(A_{0} B_{1}\right)+\left(A_{1} B_{0}\right)+\left(A_{2} B_{3}\right)-\left(A_{3} B_{2}\right)\right] \mathbf{i} \\
& +\left[\left(A_{0} B_{2}\right)-\left(A_{1} B_{3}\right)+\left(A_{2} B_{0}\right)+\left(A_{3} B_{1}\right)\right] \mathbf{j}+\left[\left(A_{0} B_{3}\right)+\left(A_{1} B_{2}\right)-\left(A_{2} B_{1}\right)+\left(A_{3} B_{0}\right)\right] \mathbf{k},[14] .
\end{aligned}
$$

Moreover, for an elliptic biquaternion $Q=A_{0}+A_{1} \mathbf{i}+A_{2} \mathbf{j}+A_{3} \mathbf{k}$, the Hamiltonian conjugate, complex conjugate and total conjugate of $Q$ are

$$
\begin{aligned}
\bar{Q} & =A_{0}-A_{1} \mathbf{i}-A_{2} \mathbf{j}-A_{3} \mathbf{k} \\
Q^{*} & =A_{0}{ }^{*}+A_{1}{ }^{*} \mathbf{i}+A_{2}{ }^{*} \mathbf{j}+A_{3}{ }^{*} \mathbf{k} \\
Q^{\dagger} & =A_{0}{ }^{*}-A_{1}{ }^{*} \mathbf{i}-A_{2}{ }^{*} \mathbf{j}-A_{3}{ }^{*} \mathbf{k}
\end{aligned}
$$

respectively. Here, superscript stars which are on $A_{0}, A_{1}, A_{2}$ and $A_{3}$ denote the usual complex conjugation. If $Q=A_{0}+A_{1} \mathbf{i}+A_{2} \mathbf{j}+A_{3} \mathbf{k}$ and $R=B_{0}+B_{1} \mathbf{i}+B_{2} \mathbf{j}+B_{3} \mathbf{k}$ are any two elliptic biquaternions then the inner product of $Q$ and $R$ is given as in the following:

$$
\langle Q, R\rangle=\frac{1}{2}(\bar{Q} R+\bar{R} Q)=\frac{1}{2}(Q \bar{R}+R \bar{Q})=A_{0} B_{0}+A_{1} B_{1}+A_{2} B_{2}+A_{3} B_{3} .
$$

The semi-norm of an elliptic biquaternion $Q=A_{0}+A_{1} \mathbf{i}+A_{2} \mathbf{j}+A_{3} \mathbf{k}$ is defined as

$$
N_{Q}=\langle Q, Q\rangle=A_{0}{ }^{2}+{A_{1}}^{2}+A_{2}{ }^{2}+{A_{3}}^{2}=Q \bar{Q}=\bar{Q} Q
$$

via aforementioned inner product. In addition, an elliptic biquaternion which has a unit real semi norm is called unit elliptic biquaternion, [14]. The set of all unit elliptic biquaternions is denoted by $\eta$ and is given as in the following

$$
\eta=\left\{Q \in H \mathbb{C}_{p}: N_{Q}=1\right\} \subset H \mathbb{C}_{p},[16] .
$$


Also, an elliptic biquaternion whose scalar part is zero called pure elliptic biquaternion, [14]. The set of all pure elliptic biquaternions whose semi-norm is equal to $|p|$ is denoted by $\zeta$ and is given as in the following

$$
\zeta=\left\{\mathbf{V}=A_{1} \mathbf{i}+A_{2} \mathbf{j}+A_{3} \mathbf{k}: N_{\mathbf{V}}=|p|, \overline{\mathbf{V}}=-\mathbf{V}, \mathbf{V}^{2}=p\right\},[16] .
$$

On the other hand, the multiplicative inverse of an elliptic biquaternion $Q$ which has a nonzero semi-norm is defined as $Q^{-1}=\frac{\bar{Q}}{N_{Q}}$. Also, for an elliptic biquaternion $Q$, its modulus is denoted by $|Q|$ and satisfies the equality $N_{Q}=|Q|^{2}$, [14].

In elliptic plane, due to [17], it is well known that the $p$ trigonometric functions; $p$-cosine (cosp) and $p$-sine (sinp) are defined as

$$
\operatorname{cosp} \theta_{p}=\cos \left(\theta_{p} \sqrt{|p|}\right), \quad \operatorname{sinp} \theta_{p}=\frac{1}{\sqrt{|p|}} \sin \left(\theta_{p} \sqrt{|p|}\right)
$$

where $\theta_{p}=\frac{1}{\sqrt{|p|}} \tan ^{-1}\left(\frac{y}{x} \sqrt{|p|}\right)$ is $p$-argument of an elliptic number $z=x+I y$. On the other hand, the functions $p$-cosine (cosp) and $p$-sine (sinp) of an elliptical complex variable $\varphi_{p}$ are given by the equalities

$$
\operatorname{cosp} \varphi_{p}=\cos \left(\varphi_{p} \sqrt{|p|}\right)=\frac{e^{I \varphi_{p}}+e^{-I \varphi_{p}}}{2}=\operatorname{cosp} x \cosh (p y)+I \operatorname{sinp} x \sinh (p y)=\sum_{n=0}^{\infty} \frac{p^{n} \varphi_{p}^{2 n}}{(2 n) !}
$$

and

$$
\operatorname{sinp} \varphi_{p}=\frac{\sin \left(\varphi_{p} \sqrt{|p|}\right)}{\sqrt{|p|}}=\frac{e^{I \varphi_{p}}-e^{-I \varphi_{p}}}{2 I}=\sin p x \cosh (p y)+I \frac{1}{p} \operatorname{cosp} x \sinh (p y)=\sum_{n=0}^{\infty} \frac{p^{n} \varphi_{p}^{2 n+1}}{(2 n+1) !}
$$

in which case, the angle $\varphi_{p}=x+I y \in \mathbb{C}_{p}$ is called as elliptical complex angle. For any two elliptical complex angles $\varphi_{p}$ and $\psi_{p}$, the following addition formulas hold [16]:

$$
\begin{aligned}
& \text { (a) } \operatorname{cosp}\left(\varphi_{p}+\psi_{p}\right)=\operatorname{cosp} \varphi_{p} \operatorname{cosp} \psi_{p}+p \operatorname{sinp} \varphi_{p} \operatorname{sinp} \psi_{p} \\
& \text { (b) } \operatorname{sinp}\left(\varphi_{p}+\psi_{p}\right)=\operatorname{sinp} \varphi_{p} \operatorname{cosp} \psi_{p}+\operatorname{cosp} \varphi_{p} \operatorname{sinp} \psi_{p}
\end{aligned}
$$

Let $Q=A_{0}+A_{1} \mathbf{i}+A_{2} \mathbf{j}+A_{3} \mathbf{k}$ be any elliptic biquaternion with a non-zero modulus while $\sqrt{{A_{1}{ }^{2}+{A_{2}}^{2}+{A_{3}}^{2}}^{2}}$ is non-zero. In this case, $Q$ can be given in the $p$-trigonometric form:

$$
Q=R\left(\operatorname{cosp} \varphi_{p}+\mathbf{w}_{Q} \operatorname{sinp} \varphi_{p}\right)
$$

with

$$
\text { (a) } R=\sqrt{A_{0}^{2}+A_{1}^{2}+A_{2}^{2}+A_{3}^{2}},(b) \operatorname{cosp} \varphi_{p}=\frac{A_{0}}{R},(c) \operatorname{sinp} \varphi_{p}=\frac{\sqrt{A_{1}^{2}+A_{2}{ }^{2}+A_{3}{ }^{2}}}{R \sqrt{|p|}},(d) \mathbf{w}_{Q}=\sqrt{|p|} \frac{A_{1} \mathbf{i}+A_{2} \mathbf{j}+A_{3} \mathbf{k}}{\sqrt{A_{1}{ }^{2}+A_{2}{ }^{2}+A_{3}{ }^{2}}}
$$

where $\varphi_{p}$ is an elliptical complex angle in the form $\varphi_{p}=x+I y \in \mathbb{C}_{p}$, [16].

Also, a natural generalization of Euler's formula for elliptic biquaternions is given as

$$
e^{\mathbf{w}_{Q} \varphi_{p}}=\operatorname{cosp} \varphi_{p}+\mathbf{w}_{Q} \operatorname{sinp} \varphi_{p}
$$

for any elliptic number $\varphi_{p}$. An elliptic biquaternion $Q=R\left(\operatorname{cosp} \varphi_{p}+\mathbf{w}_{Q} \operatorname{sinp} \varphi_{p}\right) \in H \mathbb{C}_{p}$ can be given in the elliptical exponential form as in the following:

$$
Q=R e^{\mathbf{w}_{Q} \varphi_{p}}
$$

by means of Euler's formula, [16]. Moreover, $Q$ satisfies the property which is given in the next theorem.

Theorem 2.1. For a given elliptic biquaternion $Q=R\left(\operatorname{cosp} \varphi_{p}+\mathbf{w}_{Q} \operatorname{sinp} \varphi_{p}\right)=R e^{\mathbf{w}_{Q} \varphi_{p}} \in H \mathbb{C}_{p}$, the equality

$$
Q^{n}=R^{n}\left[\operatorname{cosp}\left(n \varphi_{p}\right)+\mathbf{w}_{Q} \operatorname{sinp}\left(n \varphi_{p}\right)\right]=R^{n} e^{\mathbf{w}_{Q} n \varphi_{p}}
$$

is satisfied where $n$ is any integer, [16]. 
Lastly, we recall some information on the elliptic matrices to disambiguate the ensuing sections (See [18] for more details).

In the matrix set $M_{m \times n}\left(\mathbb{C}_{p}\right)$, which includes $m \times n$ matrices with elliptic number entries, the ordinary matrix addition and multiplication are defined. Also, the scalar multiplication is defined as

$$
\lambda A=\lambda\left(a_{i j}\right)=\left(\lambda a_{i j}\right) \in M_{m \times n}\left(\mathbb{C}_{p}\right)
$$

where $A=\left(a_{i j}\right) \in M_{m \times n}\left(\mathbb{C}_{p}\right)$ and $\lambda \in \mathbb{C}_{p}$.

For an elliptic matrix $A=\left(a_{i j}\right) \in M_{m \times n}\left(\mathbb{C}_{p}\right)$, it is known that the matrix $\bar{A}=\left(a_{i j}{ }^{*}\right) \in M_{m \times n}\left(\mathbb{C}_{p}\right)$ is the complex conjugate of $A$ where $a_{i j}{ }^{*}$ is the usual complex conjugation of $a_{i j} \in \mathbb{C}_{p}$. Also, the matrix $A^{*}=(\bar{A})^{T} \in$ $M_{n \times m}\left(\mathbb{C}_{p}\right)$ is the conjugate transpose of $A,[18]$.

\section{Hamilton Matrices of Elliptic Biquaternions}

Let $Q$ be an elliptic biquaternion. We define the linear maps

$$
\begin{aligned}
h^{+}: H \mathbb{C}_{p} & \rightarrow H \mathbb{C}_{p} & h^{-}: H \mathbb{C}_{p} & \rightarrow H \mathbb{C}_{p} \\
R & \rightarrow h^{+}(R)=Q R & , & R \rightarrow h^{-}(R)=R Q .
\end{aligned}
$$

These operators are called Hamilton operators. Using our newly defined operators and the base $\{1, \mathbf{i}, \mathbf{j}, \mathbf{k}\}$ of $H \mathbb{C}_{p}$, we can write

$$
\begin{aligned}
h^{+}(1) & =Q 1=A_{0}+A_{1} \mathbf{i}+A_{2} \mathbf{j}+A_{3} \mathbf{k} \\
h^{+}(\mathbf{i}) & =Q \mathbf{i}=-A_{1}+A_{0} \mathbf{i}+A_{3} \mathbf{j}-A_{2} \mathbf{k} \\
h^{+}(\mathbf{j}) & =Q \mathbf{j}=-A_{2}-A_{3} \mathbf{i}+A_{0} \mathbf{j}+A_{1} \mathbf{k} \\
h^{+}(\mathbf{k}) & =Q \mathbf{k}=-A_{3}+A_{2} \mathbf{i}-A_{1} \mathbf{j}+A_{0} \mathbf{k}
\end{aligned}
$$

and

$$
\begin{aligned}
h^{-}(1) & =1 Q=A_{0}+A_{1} \mathbf{i}+A_{2} \mathbf{j}+A_{3} \mathbf{k} \\
h^{-}(\mathbf{i}) & =\mathbf{i} Q=-A_{1}+A_{0} \mathbf{i}-A_{3} \mathbf{j}+A_{2} \mathbf{k} \\
h^{-}(\mathbf{j}) & =\mathbf{j} Q=-A_{2}+A_{3} \mathbf{i}+A_{0} \mathbf{j}-A_{1} \mathbf{k} \\
h^{-}(\mathbf{k}) & =\mathbf{k} Q=-A_{3}-A_{2} \mathbf{i}+A_{1} \mathbf{j}+A_{0} \mathbf{k} .
\end{aligned}
$$

Then, we find the following elliptic matrix representations

$$
H^{+}(Q)=\left(\begin{array}{cccc}
A_{0} & -A_{1} & -A_{2} & -A_{3} \\
A_{1} & A_{0} & -A_{3} & A_{2} \\
A_{2} & A_{3} & A_{0} & -A_{1} \\
A_{3} & -A_{2} & A_{1} & A_{0}
\end{array}\right)
$$

and

$$
H^{-}(Q)=\left(\begin{array}{cccc}
A_{0} & -A_{1} & -A_{2} & -A_{3} \\
A_{1} & A_{0} & A_{3} & -A_{2} \\
A_{2} & -A_{3} & A_{0} & A_{1} \\
A_{3} & A_{2} & -A_{1} & A_{0}
\end{array}\right)
$$

which correspond to Hamilton operators $h^{+}$and $h^{-}$, respectively.

By looking at an elliptic biquaternion as a four-dimensional vector, the multiplications of two elliptic biquaternions $Q=A_{0}+A_{1} \mathbf{i}+A_{2} \mathbf{j}+A_{3} \mathbf{k}$ and $R=B_{0}+B_{1} \mathbf{i}+B_{2} \mathbf{j}+B_{3} \mathbf{k}$ can be described by matrix-vector products as:

$$
Q R=H^{+}(Q) R=\left(\begin{array}{cccc}
A_{0} & -A_{1} & -A_{2} & -A_{3} \\
A_{1} & A_{0} & -A_{3} & A_{2} \\
A_{2} & A_{3} & A_{0} & -A_{1} \\
A_{3} & -A_{2} & A_{1} & A_{0}
\end{array}\right)\left(\begin{array}{c}
B_{0} \\
B_{1} \\
B_{2} \\
B_{3}
\end{array}\right)
$$


and

$$
R Q=H^{-}(Q) R=\left(\begin{array}{cccc}
A_{0} & -A_{1} & -A_{2} & -A_{3} \\
A_{1} & A_{0} & A_{3} & -A_{2} \\
A_{2} & -A_{3} & A_{0} & A_{1} \\
A_{3} & A_{2} & -A_{1} & A_{0}
\end{array}\right)\left(\begin{array}{c}
B_{0} \\
B_{1} \\
B_{2} \\
B_{3}
\end{array}\right)
$$

As already discussed up to now, the meanings of the symbols; over bar, dagger and superscript star vary according to terms which they are applied to. For the rest of the paper, we need to warn the readers about these cases.

Theorem 3.1. Let $Q=A_{0}+A_{1} \mathbf{i}+A_{2} \mathbf{j}+A_{3} \mathbf{k}, R=B_{0}+B_{1} \mathbf{i}+B_{2} \mathbf{j}+B_{3} \mathbf{k}$ be two elliptic biquaternions in the space $H \mathbb{C}_{p}$ and $\lambda$ be an elliptic number. Then the following properties hold:

(1) $Q=R \Leftrightarrow H^{+}(Q)=H^{+}(R) \Leftrightarrow H^{-}(Q)=H^{-}(R)$,

(2) $H^{+}(Q+R)=H^{+}(Q)+H^{+}(R), H^{-}(Q+R)=H^{-}(Q)+H^{-}(R)$,

(3) $H^{+}(\lambda Q)=\lambda H^{+}(Q), H^{-}(\lambda Q)=\lambda H^{-}(Q)$,

(4) $H^{+}(Q R)=H^{+}(Q) H^{+}(R), H^{-}(Q R)=H^{-}(R) H^{-}(Q)$,

(5) $H^{+}\left(Q^{-1}\right)=\left[H^{+}(Q)\right]^{-1}, H^{-}\left(Q^{-1}\right)=\left[H^{-}(Q)\right]^{-1}$,

(6) $H^{+}(\bar{Q})=\left[H^{+}(Q)\right]^{T}, H^{-}(\bar{Q})=\left[H^{-}(Q)\right]^{T}$,

(7) $H^{+}\left(Q^{*}\right)=\overline{H^{+}(Q)}, H^{-}\left(Q^{*}\right)=\overline{H^{-}(Q)}$,

(8) $H^{+}\left(Q^{\dagger}\right)=\left[H^{+}(Q)\right]^{*}, H^{-}\left(Q^{\dagger}\right)=\left[H^{-}(Q)\right]^{*}$,

(9) $\operatorname{det} H^{+}(Q)=\left(N_{Q}\right)^{2}$, $\operatorname{det} H^{-}(Q)=\left(N_{Q}\right)^{2}$, Trace $\left[H^{+}(Q)\right]=$ Trace $\left[H^{-}(Q)\right]=4 A_{0}$.

Proof. We will prove 4 and 5 the other properties can be easily shown.

(4) For convenience, let us show the components of the elliptic biquaternion $Q R$ with $A, B, C, D$. That is $Q R$ can be written in the following form:

$$
Q R=A+B \mathbf{i}+C \mathbf{j}+D \mathbf{k}
$$

where $A=A_{0} B_{0}-A_{1} B_{1}-A_{2} B_{2}-A_{3} B_{3}, B=A_{0} B_{1}+A_{1} B_{0}+A_{2} B_{3}-A_{3} B_{2}, C=A_{0} B_{2}-A_{1} B_{3}+A_{2} B_{0}+$ $A_{3} B_{1}$ and $D=A_{0} B_{3}+A_{1} B_{2}-A_{2} B_{1}+A_{3} B_{0}$. Then we obtain

$$
H^{+}(Q R)=\left(\begin{array}{cccc}
A & -B & -C & -D \\
B & A & -D & C \\
C & D & A & -B \\
D & -C & B & A
\end{array}\right)=\left(\begin{array}{cccc}
A_{0} & -A_{1} & -A_{2} & -A_{3} \\
A_{1} & A_{0} & -A_{3} & A_{2} \\
A_{2} & A_{3} & A_{0} & -A_{1} \\
A_{3} & -A_{2} & A_{1} & A_{0}
\end{array}\right)\left(\begin{array}{cccc}
B_{0} & -B_{1} & -B_{2} & -B_{3} \\
B_{1} & B_{0} & -B_{3} & B_{2} \\
B_{2} & B_{3} & B_{0} & -B_{1} \\
B_{3} & -B_{2} & B_{1} & B_{0}
\end{array}\right)=H^{+}(Q) H^{+}(R)
$$

and

$$
H^{-}(Q R)=\left(\begin{array}{cccc}
A & -B & -C & -D \\
B & A & D & -C \\
C & -D & A & B \\
D & C & -B & A
\end{array}\right)=\left(\begin{array}{cccc}
B_{0} & -B_{1} & -B_{2} & -B_{3} \\
B_{1} & B_{0} & B_{3} & -B_{2} \\
B_{2} & -B_{3} & B_{0} & B_{1} \\
B_{3} & B_{2} & -B_{1} & B_{0}
\end{array}\right)\left(\begin{array}{cccc}
A_{0} & -A_{1} & -A_{2} & -A_{3} \\
A_{1} & A_{0} & A_{3} & -A_{2} \\
A_{2} & -A_{3} & A_{0} & A_{1} \\
A_{3} & A_{2} & -A_{1} & A_{0}
\end{array}\right)=H^{-}(R) H^{-}(Q) .
$$

(5) From the inverse property, we can write

$$
Q Q^{-1}=Q^{-1} Q=1
$$

Then, the equations

$$
H^{+}(Q) H^{+}\left(Q^{-1}\right)=H^{+}\left(Q Q^{-1}\right)=H^{+}(1)=I_{4}
$$

and

$$
H^{+}\left(Q^{-1}\right) H^{+}(Q)=H^{+}\left(Q^{-1} Q\right)=H^{+}(1)=I_{4}
$$

are satisfied. It means that $H^{+}\left(Q^{-1}\right)=\left[H^{+}(Q)\right]^{-1}$. In the same manner, one can see that $H^{-}\left(Q^{-1}\right)=$ $\left[H^{-}(Q)\right]^{-1}$. 
Now we will define a function $\gamma$ from the elliptic biquaternion space $H \mathbb{C}_{p}$ to matrix space $M$ as in the following

$$
\begin{aligned}
\gamma: H \mathbb{C}_{p} & \rightarrow M \\
Q & \rightarrow \gamma(Q)=H^{+}(Q)
\end{aligned}
$$

where

$$
M=\left\{\left(\begin{array}{cccc}
A_{0} & -A_{1} & -A_{2} & -A_{3} \\
A_{1} & A_{0} & -A_{3} & A_{2} \\
A_{2} & A_{3} & A_{0} & -A_{1} \\
A_{3} & -A_{2} & A_{1} & A_{0}
\end{array}\right): A_{0}, A_{1}, A_{2}, A_{3} \in \mathbb{C}_{p}\right\}
$$

The function $\gamma$ is bijection and it comprises the following properties

$$
\begin{gathered}
\gamma(Q R)=\gamma(Q) \gamma(R) \\
\gamma(Q+R)=\gamma(Q)+\gamma(R)
\end{gathered}
$$

where $Q$ and $R$ are any elliptic biquaternions. Therefore, $\gamma$ is a linear isomorphism. But, as a consequence of theorem 3.1 (4), a map such $\delta: Q \rightarrow \delta(Q)=H^{-}(Q)$ is not a linear isomorphism. Considering the linear isomorphism $\gamma$, we call the matrix $H^{+}(Q)$ as $4 \times 4$ elliptic matrix associated with $Q$.

\section{Applications of $4 \times 4$ Elliptic Matrices Associated with the Elliptic Biquaternions}

Let $Q=R\left(\operatorname{cosp} \varphi_{p}+\mathbf{w}_{Q} \operatorname{sinp} \varphi_{p}\right)$ be an elliptic biquaternion in the $p$-trigonometric form. Since $Q$ can be written as

$$
Q=\left(R \operatorname{cosp} \varphi_{p}\right)+\left(R w_{1} \operatorname{sinp} \varphi_{p}\right) \mathbf{i}+\left(R w_{2} \operatorname{sinp} \varphi_{p}\right) \mathbf{j}+\left(R w_{3} \operatorname{sinp} \varphi_{p}\right) \mathbf{k}
$$

where $\mathbf{w}_{Q}=w_{1} \mathbf{i}+w_{2} \mathbf{j}+w_{3} \mathbf{k}$, we get the elliptic matrix $H^{+}(Q)$ in the $p$-trigonometric form as

$$
H^{+}(Q)=\left(\begin{array}{cccc}
R \operatorname{cosp} \varphi_{p} & -R w_{1} \operatorname{sinp} \varphi_{p} & -R w_{2} \operatorname{sinp} \varphi_{p} & -R w_{3} \operatorname{sinp} \varphi_{p} \\
R w_{1} \operatorname{sinp} \varphi_{p} & R \operatorname{cosp} \varphi_{p} & -R w_{3} \operatorname{sinp} \varphi_{p} & R w_{2} \operatorname{sinp} \varphi_{p} \\
R w_{2} \operatorname{sinp} \varphi_{p} & R w_{3} \operatorname{sinp} \varphi_{p} & R \operatorname{cosp} \varphi_{p} & -R w_{1} \operatorname{sinp} \varphi_{p} \\
R w_{3} \operatorname{sinp} \varphi_{p} & -R w_{2} \operatorname{sinp} \varphi_{p} & R w_{1} \operatorname{sinp} \varphi_{p} & R \operatorname{cosp} \varphi_{p}
\end{array}\right)=R\left[\operatorname{cosp} \varphi_{p} I_{4}+\operatorname{sinp} \varphi_{p} H^{+}\left(\mathbf{w}_{Q}\right)\right] .
$$

Note that

$$
H^{+}\left(\mathbf{w}_{Q}\right)=\left(\begin{array}{cccc}
0 & -w_{1} & -w_{2} & -w_{3} \\
w_{1} & 0 & -w_{3} & w_{2} \\
w_{2} & w_{3} & 0 & -w_{1} \\
w_{3} & -w_{2} & w_{1} & 0
\end{array}\right)
$$

Lemma 4.1. Let $Q=A_{0}+A_{1} \mathbf{i}+A_{2} \mathbf{j}+A_{3} \mathbf{k}=R\left(\operatorname{cosp} \varphi_{p}+\mathbf{w}_{Q} \operatorname{sinp} \varphi_{p}\right) \in H \mathbb{C}_{p}$ be given. Then, the equality $w_{1}^{2}+$ $w_{2}^{2}+w_{3}^{2}=-p$ is satisfied where $\mathbf{w}_{Q}=w_{1} \mathbf{i}+w_{2} \mathbf{j}+w_{3} \mathbf{k}$.

Proof. From (2.5)d, we know that

$$
w_{i}=\sqrt{|p|} \frac{A_{i}}{\sqrt{A_{1}^{2}+A_{2}^{2}+A_{3}^{2}}}, i=1,2,3
$$

Thus we get

$$
w_{1}^{2}+w_{2}^{2}+w_{3}^{2}=|p| \frac{A_{1}^{2}+A_{2}^{2}+A_{3}^{2}}{A_{1}^{2}+A_{2}^{2}+A_{3}^{2}}=|p|=-p .
$$

From the equation (4.1) and lemma 4.1, one immediately finds

$$
\left[H^{+}\left(\mathbf{w}_{Q}\right)\right]^{2}=p I_{4} .
$$

By means of the equalities (2.1), (2.2) and (4.2), a natural generalization of Euler's formula for matrix $H^{+}\left(\mathbf{w}_{Q}\right)$ is obtained as in the following: 


$$
\begin{aligned}
e^{H^{+}\left(\mathbf{w}_{Q}\right) \varphi_{p}} & =I_{4}+H^{+}\left(\mathbf{w}_{Q}\right) \varphi_{p}+\frac{\left(H^{+}\left(\mathbf{w}_{Q}\right) \varphi_{p}\right)^{2}}{2 !}+\frac{\left(H^{+}\left(\mathbf{w}_{Q}\right) \varphi_{p}\right)^{3}}{3 !}+\ldots \\
& =\left(\sum_{n=0}^{\infty} \frac{p^{n} \varphi_{p}^{2 n}}{(2 n) !}\right) I_{4}+\left(\sum_{n=0}^{\infty} \frac{p^{n} \varphi_{p}^{2 n+1}}{(2 n+1) !}\right) H^{+}\left(\mathbf{w}_{Q}\right) \\
& =\operatorname{cosp} \varphi_{p} I_{4}+\operatorname{sinp} \varphi_{p} H^{+}\left(\mathbf{w}_{Q}\right)
\end{aligned}
$$

for any elliptic number $\varphi_{p}$.

Theorem 4.1 (De-Moivre's formula). Let $Q=R\left(\operatorname{cosp} \varphi_{p}+\mathrm{w}_{Q} \operatorname{sinp} \varphi_{p}\right)$ be an elliptic biquaternion where $\mathbf{w}_{Q}=$ $w_{1} \mathbf{i}+w_{2} \mathbf{j}+w_{3} \mathbf{k}$. Then, the matrix $H^{+}(Q)$ and the inverse of it satisfy the following equalities

$$
\left[H^{+}(Q)\right]^{n}=R^{n}\left[\operatorname{cosp}\left(n \varphi_{p}\right) I_{4}+\operatorname{sinp}\left(n \varphi_{p}\right) H^{+}\left(\mathbf{w}_{Q}\right)\right]
$$

and

$$
\left(\left[H^{+}(Q)\right]^{-1}\right)^{n}=R^{-n}\left[\operatorname{cosp}\left(-n \varphi_{p}\right) I_{4}+\operatorname{sinp}\left(-n \varphi_{p}\right) H^{+}\left(\mathbf{w}_{Q}\right)\right]
$$

where $n$ is any positive integer, respectively.

Proof. The first part of the proof will be an induction on nonnegative integers $n$. The second part of the proof will be completed by using the first part. Suppose that the equality

$$
\left[H^{+}(Q)\right]^{k}=R^{k}\left[\operatorname{cosp}\left(k \varphi_{p}\right) I_{4}+\operatorname{sinp}\left(k \varphi_{p}\right) H^{+}\left(\mathbf{w}_{Q}\right)\right]
$$

holds. Then, we can write

$$
\left[H^{+}(Q)\right]^{k} H^{+}(Q)=R^{k}\left[\operatorname{cosp}\left(k \varphi_{p}\right) I_{4}+\operatorname{sinp}\left(k \varphi_{p}\right) H^{+}\left(\mathbf{w}_{Q}\right)\right] R\left[\operatorname{cosp} \varphi_{p} I_{4}+\operatorname{sinp} \varphi_{p} H^{+}\left(\mathbf{w}_{Q}\right)\right] .
$$

If we calculate the right side of this last equation by means of (4.2), we get

$$
\begin{aligned}
{\left[H^{+}(Q)\right]^{k+1} } & =R^{k+1}\left(\begin{array}{c}
{\left[\operatorname{cosp}\left(k \varphi_{p}\right) \operatorname{cosp} \varphi_{p}+p \sin p\left(k \varphi_{p}\right) \operatorname{sinp} \varphi_{p}\right] I_{4}} \\
+\left[\operatorname{cosp}\left(k \varphi_{p}\right) \operatorname{sinp} \varphi_{p}+\operatorname{sinp}\left(k \varphi_{p}\right) \operatorname{cosp} \varphi_{p}\right] H^{+}\left(\mathbf{w}_{Q}\right)
\end{array}\right) \\
& =R^{k+1}\left[\operatorname{cosp}\left((k+1) \varphi_{p}\right) I_{4}+\operatorname{sinp}\left((k+1) \varphi_{p}\right) H^{+}\left(\mathbf{w}_{Q}\right)\right] .
\end{aligned}
$$

This last equation completes the first part of the proof. On the other hand, from the theorem 3.1 (5), we can write

$$
\left[H^{+}(Q)\right]^{-1}=H^{+}\left(Q^{-1}\right)=H^{+}\left(R^{-1}\left[\operatorname{cosp}\left(-\varphi_{p}\right)+\mathbf{w}_{Q} \operatorname{sinp}\left(-\varphi_{p}\right)\right]\right)
$$

where $Q^{-1}=R^{-1}\left[\operatorname{cosp}\left(-\varphi_{p}\right)+\mathbf{w}_{Q} \operatorname{sinp}\left(-\varphi_{p}\right)\right]$. Thus, if we use the first part of the proof we obtain

$$
\left(\left[H^{+}(Q)\right]^{-1}\right)^{n}=R^{-n}\left[\operatorname{cosp}\left(-n \varphi_{p}\right) I_{4}+\operatorname{sinp}\left(-n \varphi_{p}\right) H^{+}\left(\mathbf{w}_{Q}\right)\right]
$$

where $n$ is any positive integer.

Example 4.1. For the elliptic biquaternion $Q=2 \sqrt{2} I+(2+2 \sqrt{2} I) \mathbf{i}+2 \sqrt{2} \mathbf{j}+(2-2 \sqrt{2} I) \mathbf{k}$ in the space $H \mathbb{C}_{-1 / 2}$, we know that it can be written in the $p$-trigonometric form as

$$
Q=2\left[\operatorname{cosp}\left(\frac{\sqrt{2}}{2} \pi-2 I \ln (1+\sqrt{2})\right)+\mathbf{w}_{Q} \operatorname{sinp}\left(\frac{\sqrt{2}}{2} \pi-2 I \ln (1+\sqrt{2})\right)\right]
$$

where $\mathbf{w}_{Q}=\frac{(1+\sqrt{2} I)}{2} \mathbf{i}+\frac{\sqrt{2}}{2} \mathbf{j}+\frac{(1-\sqrt{2} I)}{2} \mathbf{k}$ (see [16] for details). Then, we can write

such that

$$
H^{+}(Q)=2\left[\operatorname{cosp} \varphi_{p} I_{4}+\operatorname{sinp} \varphi_{p} H^{+}\left(\mathbf{w}_{Q}\right)\right]
$$

$$
\varphi_{p}=\frac{\sqrt{2}}{2} \pi-2 I \ln (1+\sqrt{2}) \text { and } H^{+}\left(\mathbf{w}_{Q}\right)=\left(\begin{array}{cccc}
0 & \frac{-1-\sqrt{2} I}{2} & -\frac{\sqrt{2}}{2} & \frac{-1+\sqrt{2} I}{2} \\
\frac{1+\sqrt{2} I}{2} & 0 & \frac{-1+\sqrt{2} I}{2} & \frac{\sqrt{2}}{2} \\
\frac{\sqrt{2}}{2} & \frac{1-\sqrt{2} I}{2} & 0 & \frac{-1-\sqrt{2} I}{2} \\
\frac{1-\sqrt{2} I}{2} & -\frac{\sqrt{2}}{2} & \frac{1+\sqrt{2} I}{2} & 0
\end{array}\right) .
$$


Every power of this matrix can be found from the theorem 4.1. For example, 13-th and 18-th powers are

and

$$
\left[H^{+}(Q)\right]^{13}=2^{13}\left[\operatorname{cosp}\left(13 \varphi_{p}\right) I_{4}+\operatorname{sinp}\left(13 \varphi_{p}\right) H^{+}\left(\mathbf{w}_{Q}\right)\right]
$$

$$
\left[H^{+}(Q)\right]^{18}=2^{18}\left[\operatorname{cosp}\left(18 \varphi_{p}\right) I_{4}+\operatorname{sinp}\left(18 \varphi_{p}\right) H^{+}\left(\mathbf{w}_{Q}\right)\right]
$$

\section{Acknowledgments}

The authors would like to thank to Mahmut Akyig̃it and Hidayet Hüda Kösal for their useful discussions. Also, the authors would like to express their sincere thanks to the anonymous referee for his/her helpful suggestions and comments which improved significantly the presentation of the paper.

\section{References}

[1] van der Waerden, B. L., Hamilton's discovery of quaternions. Math. Mag. 49 (1976), no. 5, $227-234$.

[2] Zhang, F., Quaternions and matrices of quaternions. Linear Algebra and its Applications 251 (1997), 21-57.

[3] Grob, J., Trenkler, G. and Troschke, S.-O., Quaternions: further contributions to a matrix oriented approach. Linear Algebra and its Applications 326 (2001), 205-213.

[4] Farebrother, R. W., Grob, J. and Troschke, S.-O., Matrix representation of quaternions. Linear Algebra and its Applications 362 (2003), 251-255.

[5] Cho, E., De-Moivre's formula for Quaternions. Appl. Math. Lett. 11 (1998), no. 6, 33-35.

[6] Jafari, M., Mortazaasl, H. and Yaylı, Y., De Moivre's formula for matrices of quaternions. JP Journal of Algebra, Number Theory and Applications 21 (2011), no. 1, 57-67.

[7] Hamilton, W. R., Lectures on quaternions. Hodges and Smith, Dublin, 1853.

[8] Jafari, M., On the matrix algebra of complex quaternions. Accepted for publication in TWMS Journal of Pure and Applied Mathematics (2016), DOI: $10.13140 /$ RG.2.1.3565.2321.

[9] Agrawal, O. P., Hamilton operators and dual-number-quaternions in spatial kinematics. Mech. Mach. Theory 22 (1987), no. 6, 569-575.

[10] Yayli, Y., Homothetic motions at $\mathrm{E}^{4}$. Mech. Mach. Theory 27 (1992), no. 3, 303-305.

[11] Güngör, M. A. and Sarduvan, M., A note on dual quaternions and matrices of dual quaternions. Scientia Magna 7 (2011), no. 1, 1-11.

[12] Kösal, H. H. and Tosun, M., Commutative quaternion matrices. Adv. Appl. Clifford Alg. 24 (2014), no. 3, 769-779.

[13] Akyig̃it, M., Kösal, H. H. and Tosun, M., A Note on matrix representations of split quaternions. Journal of Advanced Research in Applied Mathematics 7 (2015), no. 2, 26-39.

[14] Özen, K. E. and Tosun, M., Elliptic biquaternion algebra. AIP Conf. Proc. 1926 (2018), 020032-1-020032-6, https://doi.org/10.1063/1.5020481.

[15] Özen, K. E. and Tosun, M., A note on elliptic biquaternions. AIP Conf. Proc. 1926 (2018), 020033-1-020033-6, https://doi.org/10.1063/1.5020482.

[16] Özen, K. E. and Tosun, M., p-Trigonometric approach to elliptic biquaternions. Adv. Appl. Clifford Alg. 28:62 (2018), https:/ / doi.org/10.1007/s00006-018-0878-3.

[17] Harkin, A. A. and Harkin, J. B., Geometry of generalized complex numbers. Math. Mag. 77 (2004), no. 2, 118-129.

[18] Kösal, H. H., On commutative quaternion matrices. Sakarya University, Graduate School of Natural and Applied Sciences, Sakarya, Ph.D. Thesis, 2016.

\section{Affiliations}

KAHRAMAN ESEN ÖZEN

ADDRESS: Sakarya University, Dept. of Mathematics TR-54187 Sakarya-TURKEY.

E-MAIL: kahraman.ozen1@ogr.sakarya.edu.tr

ORCID ID : orcid.org/0000-0002-3299-6709

Murat TOSUN

ADDRESS: Sakarya University, Dept. of Mathematics TR-54187 Sakarya-TURKEY.

E-MAIL: tosun@sakarya.edu.tr

ORCID ID : orcid.org/0000-0002-4888-1412 